\title{
Differential effects of the early and late intrauterine environment on corticotrophic cell development
}

\author{
Timothy G. Butler, ${ }^{1}$ Jeff Schwartz, ${ }^{2}$ and I. Caroline McMillen ${ }^{1}$ \\ ${ }^{1}$ Department of Physiology, University of Adelaide, Adelaide, South Australia, Australia \\ 2Department of Physiology and Pharmacology, Department of Obstetrics and Gynecology, and the Perinatal Research \\ Laboratory, Bowman Gray School of Medicine, Wake Forest University, Winston-Salem, North Carolina, USA
}

\begin{abstract}
The developing embryo and fetus respond to a range of intrauterine stressors, but the effect of chronic intrauterine stress on the programmed development of pituitary corticotrophs has not been investigated. We have used a pregnant sheep model in which the embryonic environment at conception has been surgically perturbed by uterine carunclectomy. This procedure results in the development of fetuses that either are placentally restricted and chronically hypoxemic or that demonstrate compensatory placental growth and maintain normoxemia throughout late gestation. We found that uterine carunclectomy resulted in the emergence of a population of non-corticotrophin-releasing hormone (non-CRH) target cells that secreted high amounts of adrenocorticotrophic hormone (ACTH) in the fetal pituitary. This change in corticotroph development was independent of late-gestation hypoxemia. However, chronic hypoxemia during late gestation (in either carunclectomized or non-carunclectomized uterine environments) resulted in a reduction in the proportion of ACTH stored in $\mathrm{CRH}$-target. Thus, the early and late intrauterine environments differentially program the development of specific corticotrophic cell types in the fetal pituitary. These patterns of altered corticotroph development are important given the central roles of the hypothalamo-pituitary-adrenal axis in the fetal adaptive response to intrauterine stress and in the early programming of adult disease.
\end{abstract}

J. Clin. Invest. 110:783-791 (2002). doi:10.1172/JCI200215563.

\section{Introduction}

A worldwide series of epidemiological studies has demonstrated that there are significant associations between low birth weight and a range of poor adult health outcomes including high blood pressure, coronary heart disease, obesity, insulin resistance, and hypercortisolism (1-3). These associations have lead to the articulation of the "fetal origins of adult disease hypothesis," which states that fetal adaptations to a period of intrauterine deprivation result in a permanent reprogramming of key organ systems and pathophysiological outcomes in later life (2). A range of studies have implicated intrauterine glucocorticoid exposure as one of the key mediators of the effects of intrauterine deprivation and have concluded that the timing, duration, and magnitude of fetal glucocorticoid exposure may each be important in the programming of poor health outcomes (3-7). It is therefore

Received for publication March 29, 2002, and accepted in revised form July 10, 2002.

Address correspondence to: I. Caroline McMillen, Department of Physiology, University of Adelaide, Adelaide, South Australia 5005, Australia. Phone: 61-8-8303-5911; Fax: 61-8-8303-3356; E-mail: caroline.mcmillen@adelaide.edu.au.

Conflict of interest: No conflict of interest has been declared. Nonstandard abbreviations used: hypothalamo-pituitaryadrenal (HPA); adrenocorticotrophic hormone (ACTH); corticotrophin-releasing hormone (CRH); arginine vasopressin (AVP); CR, carunclectomized; NCR, non-carunclectomized; partial pressure of oxygen $\left(\mathrm{P}_{\mathrm{a}} \mathrm{O}_{2}\right)$; partial pressure of carbon dioxide $\left(\mathrm{P}_{\mathrm{a}} \mathrm{CO}_{2}\right)$; hemoglobin $(\mathrm{Hb})$. important to understand the impact of a suboptimal intrauterine environment on the functional capacity of the hypothalamo-pituitary-adrenal (HPA) axis at different stages of development. It is well established that the fetal HPA axis is activated in response to a range of acute intrauterine stressors including hypoxemia, hemorrhage, and insulin-induced hypoglycemia (8-10). The effects of chronic intrauterine stress on the fetal HPA axis are, however, less well understood. A model of chronic intrauterine stress has been developed in which the majority of the placental attachment sites, the uterine caruncles, are surgically excised from the uterus of the nonpregnant ewe $(11,12)$. During a subsequent pregnancy, a restricted number of placentomes form, and this results in a chronic placental restriction of fetal substrate supply, hypoxemia, and fetal growth restriction throughout late gestation $(11,12)$. Circulating cortisol concentrations are higher in growthrestricted fetuses of carunclectomized ewes, but, surprisingly, there is no associated increase in the fetal plasma concentrations of either immunoreactive adrenocorticotrophic hormone $(\mathrm{ACTH})$ or $\mathrm{ACTH}_{(1-39)}$, and the expression of proopiomelanocortin mRNA is decreased in the anterior pituitary of the growthrestricted fetus (13). Different subpopulations of corticotrophs, which are responsive either to corticotrophin-releasing hormone (CRH) or to arginine vasopressin (AVP) and which are differentially sensitive to negative feedback by cortisol, have been described in the adult and fetal sheep pituitary (14-17). One 
possibility is that uterine carunclectomy programs a change in the developmental characteristics of the pool of corticotroph cells within the pituitary to maintain ACTH secretion in the face of elevated cortisol concentrations during late gestation.

One important feature of uterine carunclectomy is that it does not inevitably lead to chronic fetal hypoxemia in late gestation, as there is a degree of compensatory growth of the remaining placentomes which may result in the maintenance of a relatively normoxemic, well-grown fetus $(11,12)$. Therefore, among the effects of uterine carunclectomy on the fetal HPA axis, it is possible to separate out those that may be due to a perturbation of the intrauterine environment of the early embryo from those associated with the development of chronic substrate restriction and subsequent fetal growth restriction. In the present study, we have determined whether uterine carunclectomy alters the functional heterogeneity of corticotrophic cell types within the fetal pituitary, and whether these changes are related to the perturbation of the early intrauterine environment associated with uterine carunclectomy or are solely due to the impact of a chronic restriction of fetal substrate supply in late gestation. We have determined the impact of uterine carunclectomy associated with either fetal hypoxemia or fetal normoxemia on the ACTH-secretory characteristics of pituitary corticotrophic cells and on the proportion of corticotrophic cells in the fetal anterior pituitary that are CRHresponsive. These results provide insight into the mechanisms by which perturbations of the early and late intrauterine environment may result in changes in the functional characteristics of corticotrophs in the developing pituitary.

\section{Methods}

All experiments in the study were carried out according to the guidelines of the Australian Code of Practice for the Care and Use of Animals for Scientific Procedures and approved by the Standing Committee on Ethics and Animal Experimentation at the University of Adelaide. Animals and surgery. Twenty-six pregnancy-dated Merino $\times$ Border Leicester ewes were used in this study. In 15 nonpregnant ewes (carunclectomized group; CR), the majority of visible endometrial caruncles were surgically removed under general anesthesia as previously described $(11,18)$. Briefly, with the ewe in a supine position, a low midline abdominal incision (approximately $8 \mathrm{~cm}$ ) was made to allow access to the uterus, and the majority (86-95\%) of visible caruncles were excised. After a minimum of 9 weeks' recovery from surgery, the ewes entered a mating program, and singleton pregnancies were confirmed by ultrasound at approximately 50 days of gestation. The 11 remaining ewes underwent no uterine surgery (non-carunclectomized; NCR) but were mated under the same conditions as the CR ewes.

At 98-117 days of gestation, vascular catheters were inserted into a fetal and a maternal jugular vein and/ or carotid artery and into the amniotic cavity in CR $(n=15)$ and NCR $(n=11)$ fetuses as previously described $(10,18)$. All catheters were filled with sterile heparinized saline $(500 \mathrm{IU} / \mathrm{ml}$; Multiparin; Fisons Pharmaceuticals, Pennant Hills, New South Wales, Australia) and exteriorized via an incision in the ewe's flank. The ewes were housed under a 12-hour lightdark cycle and fed once daily between two hours and six hours after lights on with water available ad libitum.

Fetal blood sample collection. Fetal arterial blood samples $(0.3 \mathrm{ml})$ were collected for the first 4 days following surgery and then three times per week until and including the day the fetuses were sacrificed. The arterial blood samples were used to measure the partial pressure of oxygen $\left(\mathrm{P}_{\mathrm{a}} \mathrm{O}_{2}\right)$ and carbon dioxide $\left(\mathrm{P}_{\mathrm{a}} \mathrm{CO}_{2}\right)$, $\mathrm{pH}$, hemoglobin content $(\mathrm{Hb})$, and oxygen saturation $\left(\mathrm{S}_{\mathrm{a}} \mathrm{O}_{2}\right)$ using an ABL 520 blood gas analyzer (Radiometer, Copenhagen, Denmark) with correction for the higher value of fetal body temperature. Fetal arterial blood samples $(2-3.5 \mathrm{ml})$ were also collected for plasma cortisol determination. Blood samples were centrifuged and plasma separated and stored at $-20^{\circ} \mathrm{C}$ for subsequent assay.

Pituitary collection and cell culture. At 138-145 days of gestation, ewes were killed with an intravenous overdose of sodium pentobarbitone $(200 \mathrm{mg} / \mathrm{kg}$; Lethobarb; Virbac PTY Ltd., Peakhurst, New South Wales, Australia). The fetal sheep were delivered via laparotomy, weighed, and killed by decapitation. Each pituitary was quickly removed and immediately placed into cold HEPES-dissociation buffer (HDB) (17). Cultured anterior pituitary cells were prepared as previously described (17) with minor modifications. Briefly, the anterior pituitary and the neurointermediate lobes of each pituitary were gently separated by blunt dissection, and the anterior pituitary tissue was subsequently minced into small fragments, washed in HDB, and placed in collagenase II (0.04\%; Worthington Biochemical Corp., Freehold, New Jersey, USA) and deoxyribonuclease I (Sigma-Aldrich, St. Louis, Missouri, USA) with gentle rocking for 2.5 hours at $37^{\circ} \mathrm{C}$. The pituitary fragments were then dispersed into cells, which were washed by repeated centrifugation and suspended in $5.0 \mathrm{ml}$ culture medium (DMEM plus Ham's F12 medium [GIBCO BRL; Life Technologies Inc., Grand Island, New York, USA] containing 10\% charcoal-stripped FCS). The cells were counted, and their viability was assessed with trypan blue. The cells were then plated in culture medium $(1 \mathrm{ml})$ at approximately $2.0 \times 10^{5}$ cells per well in 48 -well tissue culture plates (Falcon; Becton Dickinson Labware, Franklin Lakes, New Jersey, USA) and incubated at $37^{\circ} \mathrm{C}$ in a water-saturated $5 \% \mathrm{CO}_{2}$ atmosphere (BB16 Gas Incubator; Haraeus Instruments, Hanau, Germany).

Experimental protocol. Twenty-four hours after plating the cells, we treated the cells in half the wells from each pituitary with either vehicle or C-TOX, which is a synthetic hybrid molecule consisting of a $\mathrm{CRH}$ analog conjugated with the cellular toxin gelonin, at a final concentration of $25 \mathrm{nmol} / \mathrm{l}$. This treatment specifically 
eliminates CRH-target cells $(15-17,19,20)$. All cells were washed extensively in culture medium on the following day and returned to the incubator. Two days after that, the cells were again washed extensively with incubation medium (DMEM/F12 mixed 1:1; GIBCO BRL; Life Technologies Inc.) containing 0.2\% Polypep (Trace Biosciences, Castle Hill, Australia) and allowed to equilibrate to serum-free conditions for 1 hour. Cells were washed once in incubation medium and incubated for 5 hours in the absence or presence of the experimental treatments: vehicle (also termed basal; $n=26$ ), ovine CRH $\left(10^{-7} \mathrm{M} ; n=26\right)$, AVP $\left(10^{-7} \mathrm{M} ; n=23\right)$, or ovine CRH + AVP $(n=19)$ (all peptides supplied by Auspep Pty Ltd., Melbourne, Australia). After 5 hours, the culture media were collected and stored at $-20^{\circ} \mathrm{C}$ until assay. Cellular ACTH content was extracted in $0.1 \mathrm{M}$ $\mathrm{HCl}(1 \mathrm{ml})$ by repeated thawing and refreezing. The extracts were stored at $-20^{\circ} \mathrm{C}$.

ACTH RIA. The concentrations of immunoreactive ACTH in the cell culture media and cellular extracts were measured using a double-antibody RIA as previously described and validated (17). The interassay coefficient of variation was $18.3 \%$, and the intra-assay coefficient of variation was less than $10 \%$.

Cortisol RIA. Cortisol was extracted from fetal plasma using dichloromethane as previously described (13). The efficiency of recovery of ${ }^{125} \mathrm{I}$-cortisol from fetal plasma was always greater than $90 \%$. Fetal cortisol concentrations were then measured using an Orion Diagnostica RIA kit (Orion Diagnostica, Turku, Finland). The interassay coefficient of variation was less than $20 \%$, and the intra-assay coefficient of variation was less than $10 \%$.

Statistics. Fetal blood gas values are expressed as mean \pm SEM. The mean $\mathrm{P}_{\mathrm{a}} \mathrm{O}_{2}, \mathrm{P}_{\mathrm{a}} \mathrm{CO}_{2}, \mathrm{pH}, \mathrm{Hb}$, and $\mathrm{S}_{\mathrm{a}} \mathrm{O}_{2}$ were calculated for each fetus as the average of all $\mathrm{P}_{\mathrm{a}} \mathrm{O}_{2}, \mathrm{P}_{\mathrm{a}} \mathrm{CO}_{2}, \mathrm{pH}, \mathrm{Hb}$, and $\mathrm{S}_{\mathrm{a}} \mathrm{O}_{2}$ values obtained between 111 and 145 days of gestation. Arterial oxygen content $\left(\mathrm{O}_{2}\right.$ content) per $100 \mathrm{ml}$ blood $(\mathrm{ml} / \mathrm{dl})$ was calculated for each fetus with the equation $\mathrm{O}_{2}$ content $=\left(\mathrm{P}_{\mathrm{a}} \mathrm{O}_{2} \times 0.003\right)+[\mathrm{Hb}] \times\left(\mathrm{S}_{\mathrm{a}} \mathrm{O}_{2} / 100\right) \times 1.39$.

Nine of the 15 fetuses in the CR group and 4 of the 11 fetuses in the NCR group had a mean gestational $\mathrm{P}_{\mathrm{a}} \mathrm{O}_{2}$ of less than $16 \mathrm{mmHg}$, and these fetuses were categorized as being hypoxemic. The remainder of the fetuses in the CR and NCR groups were normoxemic. The separate and combined effects of carunclectomy and hypoxemia on the mean gestational values for fetal arterial blood gas and $\mathrm{pH}$ were analyzed using multifactorial ANOVA, with a Statistical Package for Social Sciences on a Vax mainframe computer (SPSSX; SPSS Inc., Chicago, Illinois, USA). Similarly, the effects of carunclectomy and hypoxemia on the gestational age profile of the fetal arterial blood gas and $\mathrm{pH}$ values were determined using a multifactorial ANOVA with carunclectomy, hypoxemia and gestational age (grouped into 5-day windows) as specified factors and repeated measures.

For the cell culture experiments, each treatment was completed at least in duplicate, and the replicate values for the measured ACTH in either the culture media (secreted) or ACTH in the cellular extracts (stored) were averaged to yield an $n=1$ for each pituitary in each treatment group. Total ACTH refers to the arithmetic sum of secreted ACTH and stored intracellular ACTH under basal conditions. All values are expressed as mean \pm SEM. The effects of carunclectomy, hypoxemia, or C-TOX on the total ACTH (per well), the amount of total ACTH secreted by the corticotrophic cells (per 104), and the absolute amount of ACTH (secreted) during the basal conditions were compared using multifactorial ANOVA (with CR vs. NCR, hypoxemia vs. normoxemia, and with vs. without the C-TOX treatment as the specified variables). The percentage of total ACTH secreted under basal conditions was also analyzed using a multifactorial ANOVA, with carunclectomy, hypoxemia, and C-TOX as the specified variables. The proportion (\%) of total ACTH present in the CRH-responsive cells (i.e., C-TOX-sensitive cells) was calculated $[(V-C y t o) / V] \times 100$, where $V=$ total ACTH in vehicle-treated wells under basal conditions, and Cyto = total ACTH in C-TOX-pretreated wells under basal conditions.

The effects of carunclectomy and hypoxemia on the proportion of ACTH stored in CRH-target cells were analyzed using a multifactorial ANOVA. The ACTHsecretory responses to $\mathrm{CRH}\left(10^{-7} \mathrm{M}\right), \mathrm{AVP}\left(10^{-7} \mathrm{M}\right)$, and $\mathrm{CRH}+\mathrm{AVP}$ were expressed as fold changes above basal ACTH secretion. The effects of carunclectomy, hypoxemia, and C-TOX on the fold changes in ACTH after $\mathrm{CRH}, \mathrm{AVP}$, and CRH + AVP stimulation were analyzed using multifactorial ANOVA.

A fetal blood sample was available from each of 17 fetal sheep during the week before death, and the separate and combined effects of carunclectomy and hypoxemia on the plasma cortisol concentrations in these animals were determined using multifactorial ANOVA. Simple linear regression analysis was also used to determine relationships between plasma cortisol concentrations and the percentage of ACTH secreted under basal conditions, the fold changes in ACTH secretion after secretagogues, and the proportion of ACTH stored in the CRH-responsive corticotrophs.

For all analyses, where the Cochrans and Bartlett-Box tests identified significant heterogeneity of variance, the data were logarithmically transformed prior to ANOVA. Where the multifactorial ANOVAs identified significant interactions between major variables, the data were split on the basis of the interactions and reanalyzed. When the ANOVAs indicated that there were differences between the groups, the Duncan's post hoc test was used to identify the significant differences $(P<0.05)$ between the mean values.

\section{Results}

Fetal outcomes. All fetuses were alive at the time of caesarean section. Carunclectomy and hypoxemia each separately resulted in a significant reduction in fetal body weight (Table 1). The $\mathrm{S}_{2} \mathrm{O}_{2}$, arterial $\mathrm{O}_{2}$ content, and $\mathrm{pH}$ were significantly lower in animals in the $\mathrm{CR}$ 
Table 1

Effect of carunclectomy and hypoxemia on fetal blood gas status and fetal body weight

\begin{tabular}{|c|c|c|c|c|c|c|c|c|}
\hline \multicolumn{2}{|c|}{ Treatment } & $\begin{array}{c}\mathrm{P}_{\mathrm{a}} \mathrm{O}_{2} \\
(\mathrm{mmHg})\end{array}$ & $\begin{array}{c}\mathrm{P}_{\mathrm{a}} \mathrm{CO}_{2} \\
(\mathrm{mmHg})\end{array}$ & $\mathrm{pH}$ & $\begin{array}{l}\mathrm{Hb} \\
(\mathrm{g} / \mathrm{dl})\end{array}$ & $\begin{array}{c}\mathrm{S}_{\mathrm{a}} \mathrm{O}_{2} \\
(\%)\end{array}$ & $\begin{array}{c}\mathrm{O}_{2} \text { content } \\
(\mathrm{ml} / \mathrm{dl})\end{array}$ & $\begin{array}{c}\text { Weight } \\
\text { (kg) }\end{array}$ \\
\hline \multirow[t]{2}{*}{ NCR } & NX & $19.3 \pm 0.9$ & $45.8 \pm 1.0$ & $7.41 \pm 0.01$ & $9.9 \pm 0.5$ & $66 \pm 3$ & $9.1 \pm 0.4$ & $4.49 \pm 0.33$ \\
\hline & $H X$ & $13.8 \pm 1.1^{\mathrm{A}}$ & $44.6 \pm 0.9$ & $7.40 \pm 0.01$ & $11.4 \pm 0.7^{\mathrm{A}}$ & $54 \pm 3^{A}$ & $8.5 \pm 0.5^{A}$ & $3.45 \pm 0.52^{\mathrm{A}}$ \\
\hline \multirow[t]{2}{*}{ CR } & NX & $19.1 \pm 0.3$ & $46.2 \pm 1.7$ & $7.39 \pm 0.01^{\mathrm{B}}$ & $10.0 \pm 0.5$ & $63 \pm 1^{B}$ & $8.8 \pm 0.4^{\mathrm{B}}$ & $4.04 \pm 0.11^{\mathrm{B}}$ \\
\hline & $\mathrm{HX}$ & $13.5 \pm 0.7^{\mathrm{A}}$ & $46.5 \pm 1.6$ & $7.38 \pm 0.01^{\mathrm{B}}$ & $12.2 \pm 0.5^{\mathrm{A}}$ & $44 \pm 3.2^{\mathrm{A}, \mathrm{B}}$ & $7.4 \pm 0.4^{\mathrm{A}, \mathrm{B}}$ & $2.75 \pm 0.38^{\mathrm{A}}$ \\
\hline
\end{tabular}

Mean gestational arterial blood gases and fetal body weight in NCR and CR groups. All values are expressed as mean \pm SEM. ASignificant difference between mean values in the normoxemic (NX) and hypoxemic (HX) groups. ${ }^{B}$ Significant difference between mean values in the NCR and CR groups.

group (i.e., CR-normoxemic and CR-hypoxemic fetuses) compared with the NCR group (i.e., NCR-normoxemic and NCR-hypoxemic fetuses) (Table 1; Figure 1). The mean gestational $\mathrm{P}_{2} \mathrm{O}_{2}, \mathrm{~S}_{2} \mathrm{O}_{2}$, and arterial $\mathrm{O}_{2}$ content were also significantly lower, and $\mathrm{Hb}$ content was significantly higher, in the hypoxemic fetuses in each group when compared with the normoxemic fetuses in the CR and NCR groups (Table 1; Figure 1).

Plasma cortisol concentrations. There were no separate or combined effects of either hypoxemia or carunclectomy on plasma cortisol concentrations, which were $90.6 \pm 31.5 \mathrm{nmol} / \mathrm{l}(n=7)$ in the hypoxemic fetuses and $45.2 \pm 21.0 \mathrm{nmol} / \mathrm{l}(n=10)$ in the normoxemic group.

Effect of carunclectomy and hypoxemia on total ACTH content. There was no significant effect of carunclectomy on the total ACTH content when expressed as either total ACTH per $10^{4}$ cells or ng ACTH per well. Cellular ACTH content measured in ng per $10^{4}$ cells was: NCR-normoxemic, $2.26 \pm 0.86$; NCR-hypoxemic, $1.34 \pm 0.36$; CR-normoxemic, $1.26 \pm 0.29$; CR-hypoxemic, $0.89 \pm 0.20$. Content in ng ACTH per well is summarized in Figure 2. There was also no effect of hypoxemia on the total ACTH present in pituitary cells in either the CR or the NCR group (Figure 2).

Effect of carunclectomy and bypoxemia on ACTH secretion. Carunclectomy had a significant effect on the rate of ACTH secretion by the fetal pituitary cells. The percentage of total ACTH that was secreted under basal conditions was significantly greater in pituitary cells from CR fetal sheep than in those from NCR fetal sheep (Table 2). Interestingly, hypoxemia itself had no effect on basal ACTH secretion in the CR and NCR groups (Table 2). There was also no significant correlation between plasma cortisol concentrations and basal ACTH secretion. The ACTH-secretory responses by intact populations of control cells (NCR, normoxemic, no C-TOX) were robust and indicative of normal, healthy in vitro responsiveness. In cells from NCR fetuses (Figure 3), CRH stimulated ACTH secretion $9.4 \pm 1.5$-fold and AVP stimulated ACTH secretion $10.6 \pm 2.0$-fold. The response to $\mathrm{CRH}$ and AVP in combination, $16.1 \pm 4.3$-fold, was significantly greater than the response to either peptide alone. Carunclectomy attenuated the ACTH-secretory responses to the hypothalamic peptides, such that the respective increases in $\mathrm{ACTH}$ secretion in the presence of CRH, AVP, or CRH + AVP were significantly lower in the CR than in the NCR group. Despite this attenuation, the responses represent a significant increase over basal ACTH secretion, and the response to CRH + AVP in combination remained significantly greater than the response to either peptide alone.

Interestingly, in these intact (no C-TOX) populations there was no separate effect of hypoxemia on the ACTH responses to the peptides in either the CR or the NCR group. There was also no significant correlation between plasma cortisol concentrations and the ACTH-secretory responses to CRH, AVP, or to CRH + AVP when the fetuses from all groups were combined.

Effect of C-TOX and hypoxemia on total ACTH content. After treatment with C-TOX, the elimination of $\mathrm{CRH}$-target cells was associated with a marked decrease in the total ACTH in the pituitary cells in both the CR and NCR groups, and there was no effect of carunclectomy on this decrease. In contrast, there was a significant interaction between the effects of C-TOX treatment and the effects of hypoxemia on the total ACTH. The relative decrease in total ACTH after C-TOX treatment was less in the hypoxemic groups (CR-hypoxemic and NCR-hypoxemic) than in the normoxemic groups (CR-normoxemic and NCR-normoxemic) (Figure 2). The proportion of ACTH contained in CRH-target cells (i.e., cells susceptible to C-TOX treatment) was therefore less in the hypoxemic than in the normoxemic fetuses in both the CR and NCR groups (Figure 4). There was no significant correlation, however, between plasma cortisol concentrations and the proportion of ACTH contained in the CRH-target cells when the CR and NCR groups were combined.

Effects of C-TOX on basal and stimulated ACTH secretion. After C-TOX treatment, there was a similar and significant increase in the fraction of the total ACTH secreted during basal conditions in the $\mathrm{CR}$ and the NCR groups, and this increase was not influenced by hypoxemia (Table 2). After elimination of the CRHtarget cells, basal ACTH secretion was significantly higher in the CR group than in the NCR group (Table 2). Importantly, there was no separate effect of hypoxemia on basal ACTH secretion after C-TOX treatment (Table 2). The increased proportion of ACTH secreted after C-TOX treatment was primarily a result of the 

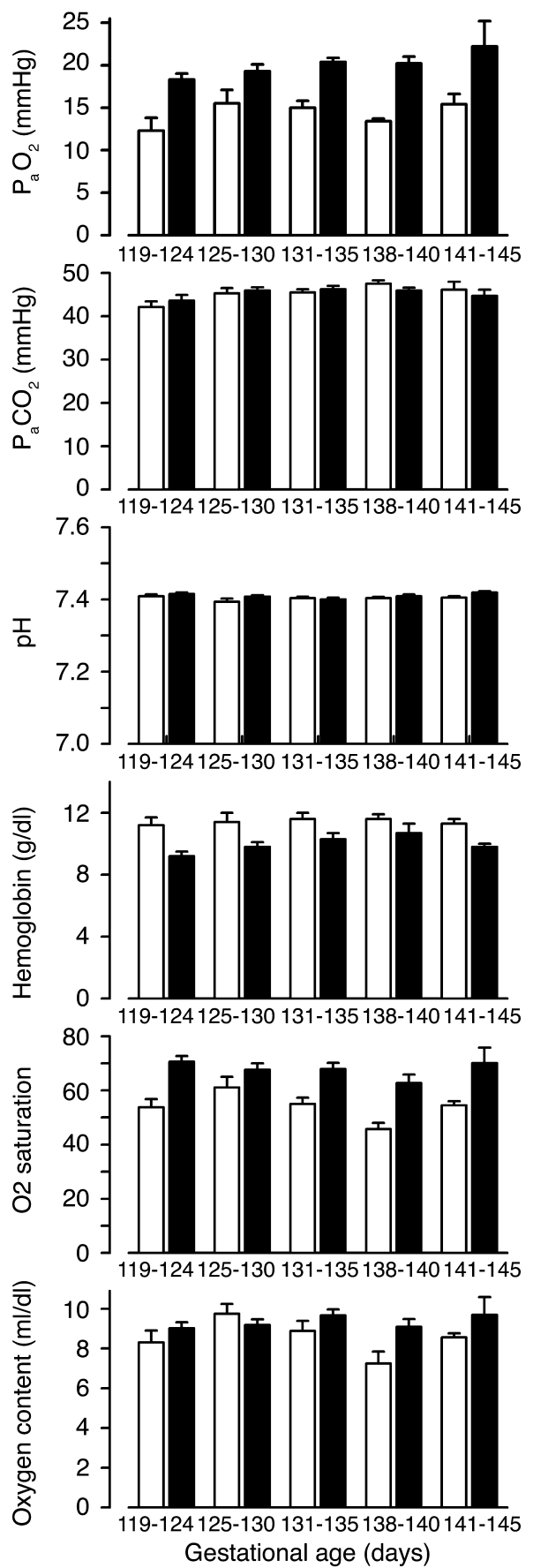
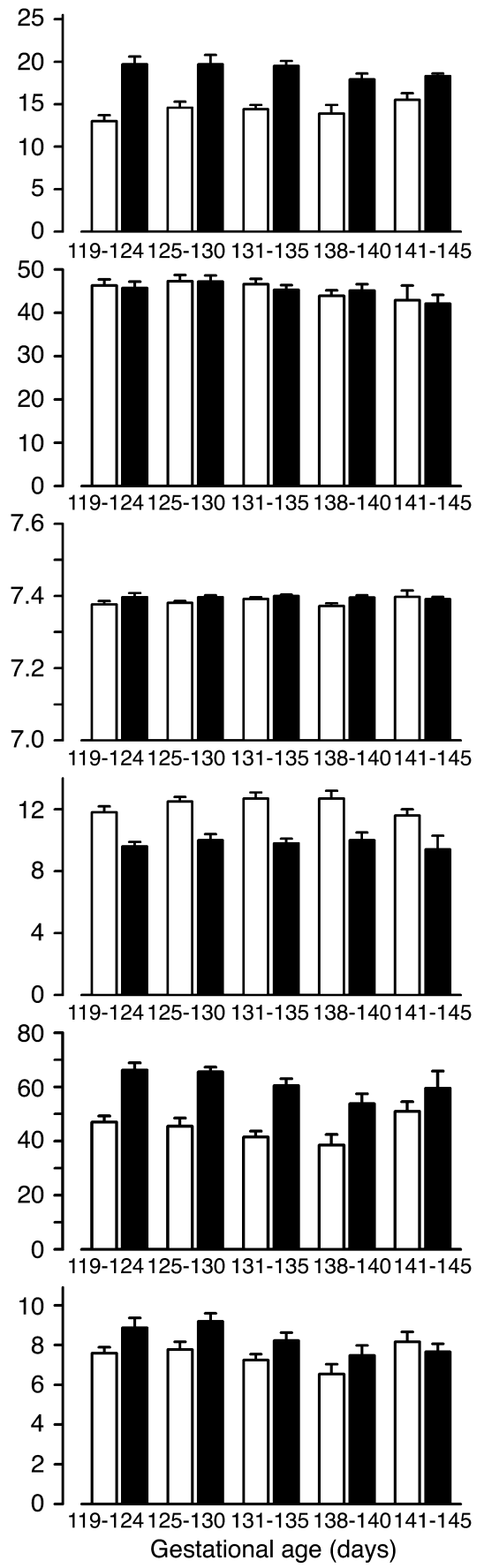

\section{Figure 1}

Mean arterial blood gases as a function of gestational age in NCR (left panels) and $C R$ (right panels) groups. Values obtained in hypoxemic fetuses are illustrated by white bars and values in normoxemic fetuses by black bars. Bars represent mean \pm SEM. Results of statistical analyses are described in Table 1. decrease in total ACTH, as C-TOX treatment did not alter the absolute amount of ACTH secreted.

Elimination of CRH-target cells significantly decreased the ACTH responses to all secretagogues. Significantly, there were differences in the effect of C-TOX treatment among the various experimental groups (Figure 5). In NCR groups, there was a small residual response to the hypothalamic peptides. Although these responses were much attenuated when compared with the corresponding ACTH responses in the intact (no C-TOX) populations, there were significant responses to $\mathrm{CRH}$ and AVP alone and a greater response to $\mathrm{CRH}+\mathrm{AVP}$ in combination. Interestingly, the presence or absence of hypoxemia made no significant difference to the fold changes in ACTH secretion. Pituitary cells from the CR fetuses, however, behaved more like cells from the adult sheep pituitary in that there was no response to $\mathrm{CRH}$ following elimination of the $\mathrm{CRH}$-target cells. In addition, there was no response to AVP alone and no effect of CRH and AVP in combination. As in the NCR group, there was no significant difference between the CR-hypoxemic and CR-normoxemic fetal pituitary cells. 


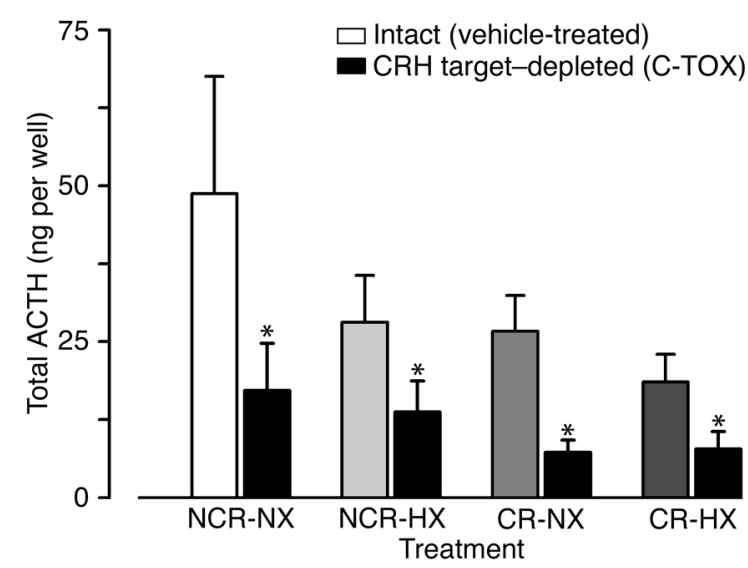

Figure 2

Total ACTH content (ng/well; mean \pm SEM) measured during basal conditions in the NCR-normoxemic (NCR-NX) and CR-NX groups and in the NCR-hypoxemic (NCR-HX) and CR-HX groups after vehicle (white bars) or C-TOX treatment (black bars). Light gray, hypoxemia; medium gray, maternal carunclectomy; dark gray, hypoxemia and maternal carunclectomy. ${ }^{*} P<0.05$, vehicle vs. C-TOX groups.

\section{Discussion}

This study has found that there are differential effects of altering the early and late intrauterine environment on the functional characteristics of the corticotroph cells in late gestation. Our results are consistent with the emergence of a population of non-CRH-target cells that secrete high amounts of ACTH in the pituitaries of fetuses that were exposed to uterine carunclectomy in early gestation. The emergence of this subpopulation of corticotrophs in the CR group during late gestation occurred whether or not these fetuses were hypoxemic throughout late gestation. Exposure to chronic hypoxemia during late gestation, however, independently resulted in a specific reduction in the proportion of ACTH stored in CRH-target cells. Thus, the ACTH-synthetic capacity of the CRHtarget cells, which do not appear to contribute to basal ACTH secretion, but which secrete ACTH in response to a superimposed acute intrauterine stress, may be diminished in the chronically hypoxemic fetus during late gestation.

Carunclectomy and fetal substrate restriction. In the current study, 9 of 15 fetuses in the CR and 4 of 11 fetuses in the NCR group were chronically hypoxemic, with mean gestational $\mathrm{P}_{\mathrm{a}} \mathrm{O}_{2}$ values of $\leq 16 \mathrm{mmHg}(21,22)$. In the remaining fetal sheep, the mean $\mathrm{P}_{\mathrm{a}} \mathrm{O}_{2}$ was over $19 \mathrm{mmHg}$, well within the reported normoxemic range $(11,13,18,21,22)$. While the mean values for fetal arterial $\mathrm{pH}$ were marginally less in the CR than in the NCR group overall, it should be noted that the arterial $\mathrm{pH}$ values in both the CR-normoxemic and the CRhypoxemic groups were well within the normal range for healthy fetal sheep in late gestation, i.e., this group was not acidemic. Fetal acidemia is therefore not a contributing factor to the changes that occurred in the ACTH-synthetic and-secretory characteristics of the corticotrophs in the CR group. It has also previously been reported that fetal sheep that are chronically hypoxemic may also be chronically hypoglycemic in late gestation (13). In a recent study on normoxemic fetuses that had either low $(<1.2 \mathrm{mmol} / \mathrm{l})$ or normal $(<1.2 \mathrm{mmol} / \mathrm{l})$ mean plasma glucose concentrations throughout late gestation, we found that chronic hypoglycemia was not associated with any differences in the basal ACTH-secretory rate, in the ACTH responses to CRH, AVP, or CRH + AVP, or in the proportion of ACTH stored in CRH-responsive corticotrophs (our unpublished observations). Thus we suggest that in the CR-hypoxemic and NCR-hypoxemic groups it is the prevailing hypoxemia during late gestation that results in a specific reduction in the proportion of ACTH stored in CRH-target cells. Furthermore, we propose that the changes in the ACTH-secretory characteristics of the corticotrophs in the CR groups are a consequence of the early intrauterine intervention, as there is no evidence for restriction of a fetal substrate present in both the CR-normoxemic and CR-hypoxemic groups.

Impact of carunclectomy on basal ACTH secretion. The percentage of $\mathrm{ACTH}$ secreted under basal conditions was greater in the CR than in the NCR fetuses, and this effect was independent of the prevailing fetal $\mathrm{P}_{\mathrm{a}} \mathrm{O}_{2}$. As argued above, it is therefore unlikely that this increase in basal ACTH secretion is related to the effects of placental restriction experienced by fetuses in vivo in late gestation in the $\mathrm{CR}$ group. Interestingly, the higher basal output of ACTH was maintained in the CR group after C-TOX treatment, reflecting maintained ACTH secretion despite a decrease in total ACTH content.

Several groups, including ours, have described functional heterogeneity of corticotrophs on the basis of responses to specific hypothalamic peptides, including CRH and AVP $(14-17,23)$. In adult sheep anterior pituitaries, treatment with C-TOX eliminates the response to CRH, while basal ACTH secretion is maintained, or increased, and a residual response to AVP remains (14, 15). On this basis it has been concluded that there are at least two populations of corticotrophs, one responsive to CRH and AVP (and susceptible to C-TOX), and one that secretes the majority of ACTH under basal conditions and that responds to AVP but not CRH.

\section{Table 2}

The percentage of total ACTH secreted under basal conditions in the presence of either vehicle or C-TOX

\begin{tabular}{cccc}
\hline \multicolumn{2}{c}{ Treatment } & Vehicle & C-TOX \\
NCR & Normoxemic & $1.52 \pm 0.31$ & $5.02 \pm 0.96^{\mathrm{B}}$ \\
& Hypoxemic & $1.85 \pm 0.24$ & $4.21 \pm 0.65^{\mathrm{B}}$ \\
$\mathrm{CR}$ & Normoxemic & $2.55 \pm 0.61^{\mathrm{A}}$ & $5.78 \pm 1.35^{\mathrm{A}, \mathrm{B}}$ \\
& Hypoxemic & $5.30 \pm 1.52^{\mathrm{A}}$ & $8.38 \pm 2.31^{\mathrm{A}, \mathrm{B}}$
\end{tabular}

The percentage of total ACTH secreted under basal conditions in the presence of vehicle or C-TOX. All values are expressed as mean \pm SEM. ${ }^{A}$ Significant difference between mean values in the NCR and CR groups. BSignificant difference between vehicle and C-TOX treatment. 


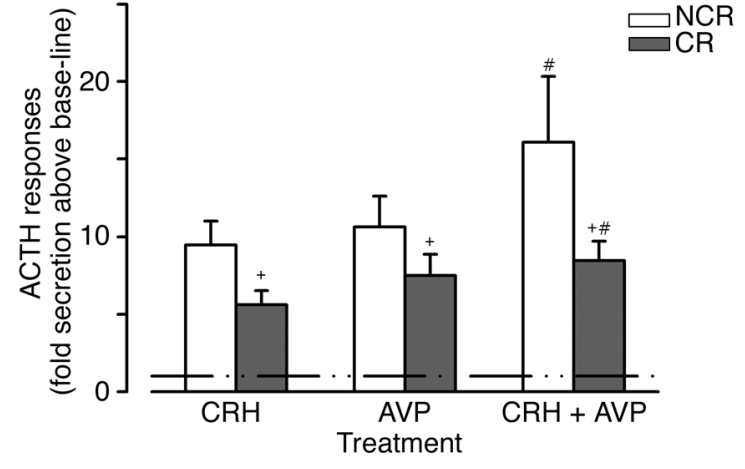

Figure 3

Fold change in $\mathrm{ACTH}$ secretion (mean $\pm \mathrm{SEM}$ ) after $\mathrm{CRH}$, AVP, or $\mathrm{CRH}$ + AVP administration in the NCR (white bars) and CR (gray bars) groups in the absence of C-TOX treatment. The horizontal dashed line denotes no increase in secretion over basal rates. ${ }^{+} P<0.05$, CR vs. NCR groups; ${ }^{\#} P<0.05, \mathrm{CRH}+\mathrm{AVP}$ vs. CRH or AVP alone.

A similar conclusion had been reached in studies using the reverse hemolytic plaque assay in anterior pituitary cells from the adult rat (24). The maintenance of basal ACTH secretion after treatment with C-TOX is consistent with findings from previous studies on anterior pituitary cells from the fetal sheep, which have described the presence of at least one type of corticotroph that accounts for basal ACTH secretion and is resistant to C-TOX pretreatment (17).

In the current study, there was no difference in the ACTH-secretory responses to AVP alone or in combination with $\mathrm{CRH}$ in the CR group after C-TOX treatment. This is in contrast to the NCR group, where the ACTH responses to AVP in combination with CRH were greater than those to $\mathrm{CRH}$ alone after C-TOX treatment. This implies that the ACTH response to AVP in the pituitaries of CR fetuses in the absence of C-TOX treatment is derived from cells that are responsive to both AVP and CRH. While previous studies with C-TOX in pituitary cells from adult sheep have not provided evidence for a population of corticotrophs that secretes ACTH under basal conditions but does not respond to AVP, such cells have been described in studies using the reverse hemolytic plaque assay in adult rat anterior pituitary cells (23).

In the present study, pituitary cells from normoxemic NCR fetuses had levels of basal ACTH secretion similar to those measured in previous studies on pituitary cells collected from healthy, term fetal sheep (17). Consistent with previously observed effects of C-TOX (17), pituitaries from normoxemic NCR and $\mathrm{CR}$ fetuses in the present studies showed an increase in basal ACTH secretion in the normoxemic NCR group after C-TOX treatment.

Interestingly, the ACTH responses to $\mathrm{CRH}$ and to $\mathrm{CRH}+\mathrm{AVP}$ in the NCR group were higher after C-TOX than in the CR group. This contrasts with previous studies with C-TOX in adult ovine pituitary cells, in which there was no response to $\mathrm{CRH}$, either alone or in combination with AVP, in the CRH-target-depleted populations. One explanation is that the C-TOX was ineffective in eliminating CRH-target cells. This would not appear to be the case, since pituitaries removed from NCR and CR fetuses were treated identically with different effects in the two groups. Another explanation for these findings is that in the healthy developing pituitary there is increased functional plasticity, such that cells that do not have $\mathrm{CRH}$ receptors at the time of treatment with C-TOX and are therefore not susceptible to the toxin survive the treatment and subsequently become CRH-target corticotrophs. These surviving cells might be characterized as normally secreting ACTH under basal conditions, but they only develop CRH receptors and CRH sensitivity in culture. Alternatively, there might be some type of multipotential or stem cell that becomes a corticotroph after the treatment with C-TOX (24). We have shown in rat corticotrophs that a limited response to $\mathrm{CRH}$ reemerges at around 3 days after C-TOX treatment (25). In addition, Jia and coworkers have described the emergence of a new population of $\mathrm{CRH}$-responsive corticotrophs following the elimination of the existing $\mathrm{CRH}$-target cells by photoablation (24). Although the reemergence of an ACTH response to CRH has not been observed previously in ovine cells, it should be noted that the developmental plasticity of fetal ovine pituitary cells is likely to be higher than that of adult cells. During development, the turnover rate for corticotrophs is extremely high and is probably determined by mitosis and apoptosis as well as differentiation $(26,27)$. Numerous examples exist of pituitary plasticity involving melanotrophs, somatotrophs, lactotrophs, and gonadotrophs, in which there is interchange or transdifferentiation in the course of normal physiological adjustments (28-31). It is particularly noteworthy that the greater response to CRH after C-TOX treatment is a characteristic of pituitary cells from NCR, rather than CR, fetuses. This

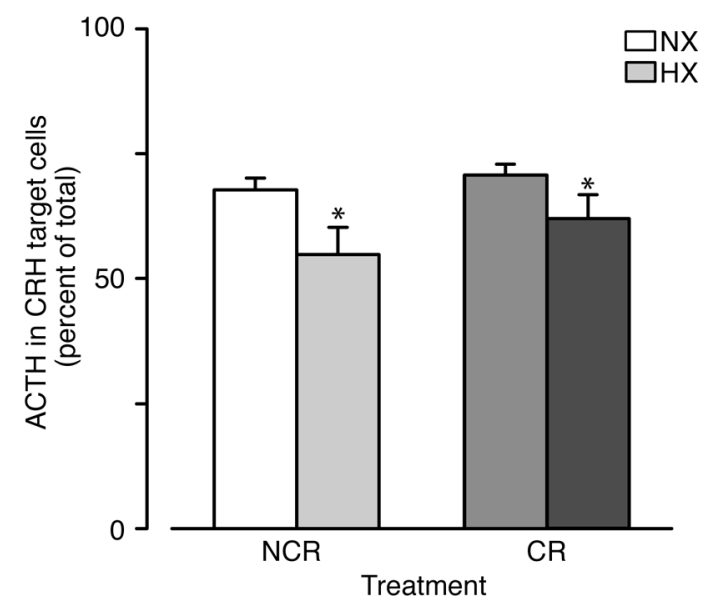

Figure 4

The proportion of total ACTH (mean \pm SEM) present in CRH-target cells in the NCR and CR groups. Light gray, hypoxemia; medium gray, maternal carunclectomy; dark gray, hypoxemia and maternal carunclectomy. ${ }^{*} P<0.05, \mathrm{HX}$ versus $\mathrm{NX}$. 

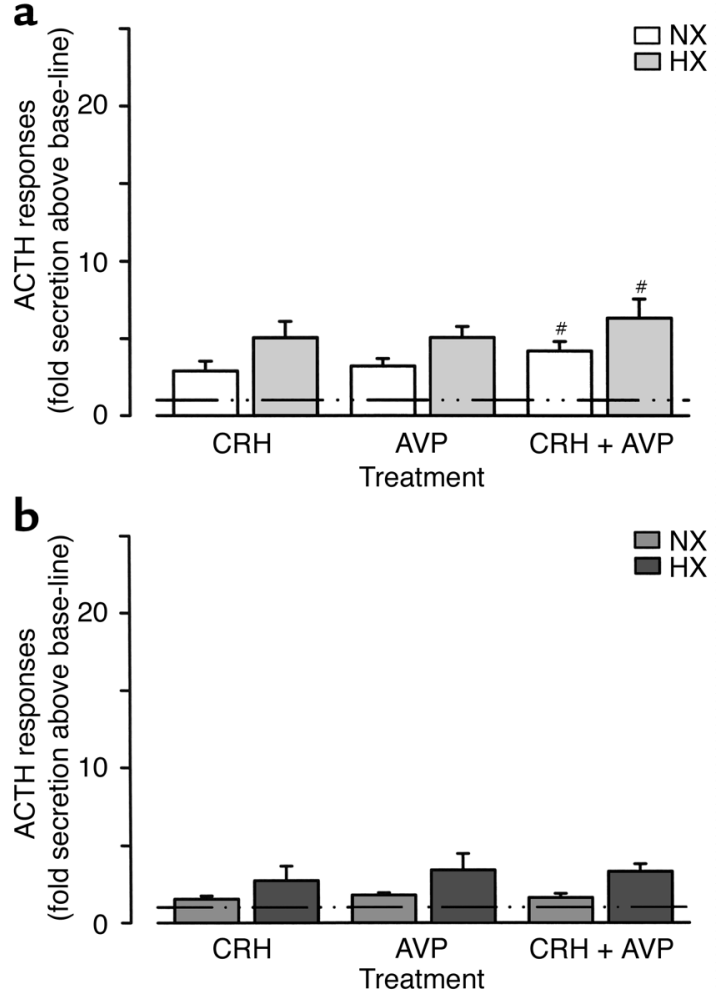

Figure 5

(a) Fold change in ACTH secretion (mean \pm SEM) after $C R H, A V P$, or CRH + AVP administration in NCR after C-TOX treatment. Light gray, hypoxemia. ${ }^{\#} P<0.05, \mathrm{CRH}+\mathrm{AVP}$ vs. $\mathrm{CRH}$ or AVP alone. (b) Fold change in ACTH secretion (mean \pm SEM) after $C R H$, AVP, or $\mathrm{CRH}+$ AVP administration in CR groups after C-TOX treatment. Medium gray, maternal carunclectomy; dark gray, hypoxemia and maternal carunclectomy.

suggests that the plasticity of the fetal pituitary, apparently characteristic of the control fetuses, is lacking in CR fetuses. Whether or not CR specifically has an effect on multipotential pituitary cells or transdifferentiation of other cells remains an interesting question.

Impact of carunclectomy on corticotrophic cell types in the fetal pituitary in late gestation. While the basal output of ACTH was greater in the CR group, there was no difference in the proportion of ACTH present in CRH-target cells between the CR and NCR groups. The proportion of ACTH stored in CRH-responsive cells (i.e., C-TOX-sensitive cells) was around $70 \%$ of the total ACTH content in pituitaries from normoxemic fetal sheep in both the CR and NCR groups, consistent with CRH-responsive cells of the pituitary of the healthy, term sheep fetus (17). The simplest explanation of these results is that carunclectomy results in the development of a population of non-CRH-target corticotrophs that secrete high amounts of ACTH during basal conditions but does not alter the ACTH content of the population of the CRH-target cells. Recent studies have found that nutritional or hormonal manipulation of the intrauterine environment or of the embryo during the preimplantation period results in alterations in fetal growth patterns, fetal HPA function, and gestation length (32-34). Thus the reprogramming of early pituitary development may be a key response to perturbations of the interaction between the uterine endometrium and the developing embryo.

Impact of hypoxemia on corticotrophic cell types. Chronic hypoxemia was associated with a decrease in the amount of ACTH stored in CRH-responsive cells and with a trend toward the reemergence of $\mathrm{CRH}$ sensitivity after C-TOX treatment. This effect was present to a greater extent in the NCR group. It has been demonstrated that fetal plasma cortisol concentrations are higher in the chronically hypoxemic fetus in late gestation $(13,21)$. Furthermore, cortisol can act to suppress ACTH synthesis in CRH-target corticotrophs $(15,17)$. In the present study, however, while there was a trend toward higher circulating cortisol concentrations in the hypoxemic fetuses, we found no evidence for an inverse correlation between circulating cortisol and the proportion of ACTH stored in the $\mathrm{CRH}$-responsive cells in the pituitaries from the fetal sheep in the NCR and CR groups. Alternatively, the effect of chronic hypoxemia on the amount of ACTH stored in the CRH-target cells may reflect a prior history of hypothalamic stimulation in vivo as a consequence of the low $\mathrm{P}_{\mathrm{a}} \mathrm{O}_{2}$.

Summary. In summary, our results are consistent with the emergence of a population of non-CRH-target cells that secrete high amounts of ACTH in the pituitaries of fetuses that were exposed to the CR uterine environment in early gestation. This may represent a reprogramming of the pattern of corticotroph development to ensure that fetal ACTH secretion can be maintained throughout late gestation independently of whether placental restriction of fetal substrate supply subsequently ensues. Given that altered corticotroph development persists in otherwise metabolically healthy fetuses in the CR group for up to 5 months after the early intrauterine intervention, it may also persist after birth to result in a maintained ACTH response to postnatal stressors. In this context, it is interesting that children and adults with lower birth weights have been reported to excrete more cortisol or its metabolites in urine $(4,35)$. Furthermore a study on adult men found that low birth weight and/or features of the metabolic syndrome were associated with measures of increased activity of the HPA axis (3). Our study provides one potential mechanism to explain the associations between low birth weight, increased adult HPA activity, and the metabolic syndrome.

While exposure to chronic hypoxemia during late gestation did not appear to alter the subpopulations of corticotroph cell types present within the fetal pituitary, it did result in a specific reduction in the proportion of ACTH stored in CRH-target cells. Thus, the ACTH-synthetic capacity of the $\mathrm{CRH}$-target cells, which do not appear to contribute to basal ACTH secretion, but which secrete ACTH in response to a superimposed acute intrauterine stress, may be 
relatively diminished in the chronically hypoxemic fetus during late gestation. It has previously been reported that there is a decrease in pituitary proopiomelanocortin mRNA levels, while basal circulating immunoreactive ACTH concentrations are maintained in the hypoxemic CR fetus in late gestation (13). Thus it is possible that circulating ACTH levels are maintained as a consequence of secretion from non- $\mathrm{CRH}-$ target corticotrophs while the expression of proopiomelanocortin is suppressed in the CRH-target cells. These patterns of altered corticotroph development are important given the central roles of the HPA axis in the fetal adaptive response to intrauterine stress and in the early programming of adult disease.

\section{Acknowledgments}

This work was funded by the National Health and Medical Research Council. The authors gratefully acknowledge the expert assistance of Michael Adams, Frank Carbone, Lisa Edwards, Anne Jurisevic, Jacob Ross, and Kirsty Warnes with the animal experiments.

1. Barker, D.J.P. 1992. Fetal and infant origins of adult disease. British Medical Journal. London, United Kingdom. 343 pp.

2. Barker, D.J.P. 1998. Mothers, babies and health in later life. Churchill Livingstone. Edinburgh, United Kingdom. 217 pp.

3. Reynolds, R.M., et al. 2002. Altered control of cortisol secretion in adult men with low birth weight and cardiovascular risk factors. J. Clin. Endocrinol. Metab. 86:245-250.

4. Langley-Evans, S.C., Phillips, G.J., Gardner, D.S., and Jackson, A.A. 1996. Role of glucocorticoids in programming of maternal diet-induced hypertension in the rat. J. Nutr. Biochem. 7:173-178.

5. Levitt, N.S., Lindsay, R.S., Holmes, M.C., and Seckl, J.R. 1996. Dexamethasone in the last week of pregnancy attenuates hippocampal glucocorticoid receptor gene expression and elevates blood pressure in the adult offspring in the rat. Neuroendocrinology. 64:412-418.

6. Edwards, L.J., and McMillen, I.C. 2001. Maternal undernutrition increases arterial blood pressure in the sheep fetus during late gestation. J. Physiol. 533:561-570.

7. Dodic, M., Peers, A., Coghlan, J.P., and Wintour, M. 1999. Can excess glucocorticoid predispose to cardiovascular and metabolic disease in middle age? Trends Endocrinol. Metab. 110:86-91.

8. Gardner, D.S., Fletcher, A.J., Fowden, A.L., and Giussani, D.A. 2001. Plasma adrenocorticotropin and cortisol concentrations during acute hypoxemia after a reversible period of adverse intrauterine conditions in the ovine fetus during late gestation. Endocrinology. 142:589-598.

9. Coulter, C.L., Martin, M.C., Voytek, C.C., Hofmann, J.I., and Jaffe, R.B. 1993. Response to hemorrhagic stress in the rhesus monkey fetus in utero: effects on the pituitary-adrenal axis. J. Clin. Endocrinol. Metab. 76:1234-1240.

10. Edwards, L.J., et al. 2001. Responses of the fetal pituitary-adrenal axis to acute and chronic hypoglycemia during late gestation in the sheep. Endocrinology. 142:1778-1785.

11. Robinson, J.S., Kingston, E.J., Jones, C.T., and Thorburn, G.D. 1979. Studies on experimental growth retardation in sheep. The effect of removal of endometrial caruncles on fetal size and metabolism. J. Dev. Physiol. 1:379-398.

12. McMillen, I.C., et al. 2001. Fetal growth restriction: adaptations and consequences. Reproduction. 122:195-204.

13. Phillips, I.D., et al. 1996. Placental restriction alters the functional development of the pituitary-adrenal axis in the sheep fetus during late gestation. Pediatr. Res. 40:861-866.

14. van de Pavert, S.A., Clarke, I.J., Rao, A., Vrana, K.E., and Schwartz, J. 1997. Effects of vasopressin and elimination of corticotropin-releasing hormone-target cells on pro-opiomelanocortin mRNA levels and adrenocorticotropin secretion in ovine anterior pituitary cells. J. Endocrinol. 154:139-147.

15. Schwartz, J., et al. 1994. Secretion of adrenocorticotrophin (ACTH) and ACTH precursors in ovine anterior pituitary cells: actions of corticotrophin-releasing hormone, arginine vasopressin and glucocorticoids. J. Endocrinol. 140:189-195.

16. Perez, F.M., Schwartz, J., and Rose, J.C. 1997. Developmental changes in ovine corticotrophs in vitro. Endocrinology. 138:916-921.

17. Butler, T.G., Schwartz, J., and McMillen, I.C. 1999. Functional heterogeneity of corticotrophs in the anterior pituitary of the sheep fetus. J. Physiol. 516:907-913.

18. Edwards, L.J., Simonetta, G., Owens, J.A., Robinson, J.S., and McMillen, I.C. 1999. Restriction of placental and fetal growth in sheep alters fetal blood pressure responses to angiotensin II and captopril. J. Physiol. 515:897-904.

19. Schwartz, J., and Vale, W. 1988. Dissociation of the adrenocorticotropin secretory responses to corticotropin-releasing factor (CRF) and vasopressin or oxytocin by using a specific cytotoxic analog of CRF. Endocrinology. 122:1695-1700.

20. Schwartz, J., Penke, B., Rivier, J., and Vale, W. 1987. A new cytotoxin specific for the target cells of corticotropin-releasing factor. Endocrinology. 121:1454-1461.

21. Gardner, D.S., Fletcher, A.J.W., Bloomfield, M.R., Fowden, A.L., and Giussani, D.A. 2002. Effects of prevailing hypoxaemia, acidaemia or hypoglycaemia upon the cardiovascular, endocrine and metabolic responses to acute hypoxaemia in the ovine fetus. J. Physiol. 540:351-366.

22. Char, V.C., and Creasy, R.K. 1977. Glucose and oxygen metabolism in normally oxygenated and spontaneously hypoxemic fetal lambs. Am.J. Obstet. Gynecol. 127:499-504.

23. Jia, L.G., Canny, B.J., Orth, D.N., and Leong, D.A. 1991. Distinct classes of corticotropes mediate corticotropin-releasing hormone- and arginine vasopressin-stimulated adrenocorticotropin release. Endocrinology. 128:197-203.

24. Jia, L.G., Canny, B.J., and Leong, D.A. 1992. Paracrine communication regulates adrenocorticotropin secretion. Endocrinology. 130:534-539.

25. Schwartz, J., Familari, M., Wallace, C., and Funder, J.W. 1989. Dissociation of ACTH-secretory mechanisms in rat pituitary cells: evidence that basal and vasopressin-stimulated secretion act via a mechanism distinct from that of corticotropin-releasing factor. J. Neuroendocrinol. 1:117-120.

26. Taniguchi, Y., Kominami, R., Yasutaka, S., and Kawarai, Y. 2000. Proliferation and differentiation of pituitary corticotrophs during the fetal and postnatal period: a quantitative immunochemical study. Anat. Embryol. (Berl.) 201:229-234.

27. Levy, A. 1999. Mitosis and apoptosis in the pituitary gland: tumour formation or hyperplasia. Baillières Clin. Endocrinol. Metab. 13:353-365.

28. Schwartz, J. 2000. Intercellular communication in the anterior pituitary. Endocr. Rev. 21:488-513.

29. Vazquez-Martinez, R., et al. 2001. Melanotrope cell plasticity: a key mechanism for the physiological adaptation to background color changes. Endocrinology. 142:3060-3067.

30. Goth, M.I., Lyons, C.E., Ellwood, M.R., Barrett, J.R., and Thorner, M.O. 1996. Chronic estrogen treatment in male rats reveals mammosomatotropes and allows inhibition of prolactin secretion by somatostatin. Endocrinology. 137:274-280.

31. Childs, G.V. 2000. Growth hormone cells as co-gonadotropes: partners in the regulation of the reproductive system. Trends Endocrinol. Metab. 11:168-175.

32. Kleemann, D.O., Walker, S.K., and Seamark, R.F. 1994. Enhanced fetal growth in sheep administered progesterone during the first three days of pregnancy. J. Reprod. Fertil. 102:411-417.

33. Kwong, W.Y., Wild, A.E., Roberts, P., Willis, A.C., and Fleming, T.P. 2000. Maternal undernutrition during the preimplantation period of rat development causes blastocyst abnormalities and programming of postnatal hypertension. Development. 127:4195-4202.

34. Edwards, L.J., and McMillen, I.C. 2002. Impact of maternal undernutrition during the periconceptional period, fetal number and fetal sex on the development of the hypothalamo-pituitary-adrenal axis in sheep during late gestation. Biol. Reprod. 66:1562-1569.

35. Clark, P.M., et al. 1996. Size at birth and adrenocortical function in childhood. Clin. Endocrinol. (Oxf.) 45:721-726. 